\title{
A!
}

This is an electronic reprint of the original article.

This reprint may differ from the original in pagination and typographic detail.

Gasparinetti, S.; Solinas, P.; Yoon, Y.; Pekola, J.P.

\section{Single Cooper-pair pumping in the adiabatic limit and beyond}

Published in:

Physical Review B

DOI:

10.1103/PhysRevB.86.060502

Published: 02/08/2012

Document Version

Publisher's PDF, also known as Version of record

Please cite the original version:

Gasparinetti, S., Solinas, P., Yoon, Y., \& Pekola, J. P. (2012). Single Cooper-pair pumping in the adiabatic limit and beyond. Physical Review B, 86(6), 1-5. [060502]. https://doi.org/10.1103/PhysRevB.86.060502

This material is protected by copyright and other intellectual property rights, and duplication or sale of all or part of any of the repository collections is not permitted, except that material may be duplicated by you for your research use or educational purposes in electronic or print form. You must obtain permission for any other use. Electronic or print copies may not be offered, whether for sale or otherwise to anyone who is not an authorised user. 


\title{
Single Cooper-pair pumping in the adiabatic limit and beyond
}

\author{
S. Gasparinetti, ${ }^{1, *}$ P. Solinas, ${ }^{1,2}$ Y. Yoon, ${ }^{1}$ and J. P. Pekola ${ }^{1}$ \\ ${ }^{1}$ Low Temperature Laboratory (OVLL), Aalto University, P.O. Box 15100, FI-00076 Aalto, Finland \\ ${ }^{2}$ COMP Centre of Excellence, Department of Applied Physics, Aalto University, P.O. Box 11100, FI-00076 Aalto, Finland
}

(Received 1 June 2012; published 2 August 2012)

\begin{abstract}
We demonstrate controlled pumping of Cooper pairs down to the level of a single pair per cycle, using an rf-driven Cooper-pair sluice. We also investigate the breakdown of the adiabatic dynamics in two different ways. By transferring many Cooper pairs at a time, we observe a crossover between pure Cooper-pair and mixed Cooper-pair-quasiparticle transport. By tuning the Josephson coupling that governs Cooper-pair tunneling, we characterize Landau-Zener transitions in our device. Our data are quantitatively accounted for by a simple model including decoherence effects.
\end{abstract}

DOI: 10.1103/PhysRevB.86.060502

PACS number(s): 74.50.+r, 75.45.+j, 85.25.Cp, 03.65.Yz

Charge pumps ${ }^{1-5}$ and turnstiles ${ }^{6}$ have recently attracted considerable attention. They could be used as building blocks for quantum computing devices, ${ }^{7}$ or to create a quantized current source that would pair up with the Josephson voltage and quantum Hall resistance to close the so-called quantum metrology triangle. ${ }^{8}$ Among different types of realizations, Cooper-pair pumps ${ }^{9-12}$ stand out as macroscopically coherent objects, with the phase of the superconducting order parameter in the leads playing a key role. In addition, the cyclic path is described in the space of parameters when pumping is equipped with a nontrivial geometric structure, allowing for the observation of geometric-phase effects. ${ }^{13}$ In the adiabatic limit, a general relation was derived ${ }^{14}$ connecting the pumped charge to the geometric (Berry) phase accumulated by the system ground state along a pumping cycle. This relation was experimentally demonstrated in Ref. 15. Beyond the adiabatic limit, we have recently proposed to employ a Cooper-pair pump as a Landau-Zener interferometer for geometric phases. ${ }^{16}$

In this Rapid Communication, we demonstrate controlled pumping of a single Cooper pair, using an rf-driven Cooperpair sluice. ${ }^{10,17}$ Accessing this regime opens attractive possibilities for Cooper-pair pumping, from quantum metrology to the study of dissipation in driven quantum systems, ${ }^{18}$ also in connection with geometric phases. We then investigate the breakdown of adiabatic pumping. In the sluice, this is expected to take place via Landau-Zener transitions (LZTs) at level anticrossings. We reach the nonadiabatic limit in two different ways. By pumping many Cooper pairs at a time, we witness a crossover between pure Cooper-pair and mixed Cooperpair-quasiparticle dynamics, due to the continuous generation of nonequilibrium quasiparticles by the nonadiabatic drive. By tuning the Josephson coupling that governs Cooper-pair tunneling, we characterize LZTs in our device. Our data are quantitatively accounted for by a simple model comprising LZTs and realistic decoherence.

The Cooper-pair sluice (Fig. 1) is a fully tunable Cooperpair transistor, consisting of a small superconducting island connected to leads by two superconducting quantum interference devices (SQUIDs). The SQUIDs are controlled independently by inductively coupled on-chip coils, so that they can serve as Josephson junctions of tunable energy $J_{1}, J_{2}$. A gate electrode capacitively coupled to the island induces a polarization charge $n_{g}=C_{g} V_{g} / 2 e$ in units of Cooper pairs, where $V_{g}$ is the gate voltage and $C_{g}$ the cross capacitance between the gate and the island. The device is typically operated in the charging regime, meaning that $4 E_{C} \gg J_{1}, J_{2}$ ( $E_{C}=e^{2} / 2 C_{\Sigma}$ is the single-electron charging energy of the island, $C_{\Sigma}$ being its total capacitance). Pumping is realized by steering the three control parameters $J_{1}, J_{2}$, and $n_{g}$ in a periodic fashion. The gate is used as a piston to change the number of Cooper pairs on the island, while the SQUIDs are operated as valves so as to impart a direction to the flow of charge. A typical pumping cycle is described in Fig. 1(c).

We fabricate the devices by standard electron-beam lithography, two-angle Al evaporation, and liftoff. Small Josephson junctions (area $\approx 70 \times 70 \mathrm{~nm}$ ) are obtained by oxidization of the first $\mathrm{Al}$ layer in a controlled $\mathrm{O}_{2}$ atmosphere. A scanning electron micrograph of a representative device is shown in Fig. 1(b). From the Coulomb-blockade conductance peak measured at $2 \mathrm{~K}$ we obtain $E_{C}=0.77 \mathrm{~K}$. The normal-state resistance of the device at $2 \mathrm{~K}$ is $R_{N}=29 \mathrm{k} \Omega$. Using the measured superconducting gap at a base temperature $\Delta=$ $180 \mu \mathrm{eV}, R_{N}$, and the Ambegaokar-Baratoff formula, we estimate a maximum Josephson energy $J_{\max }^{0}=0.46 \mathrm{~K}$ per SQUID. From switching statistics to the normal state in a current-biased configuration, we estimate the ratio between the maximum and minimum Josephson couplings obtained by varying the flux to be $J_{\min }^{0} / J_{\max }^{0} \leqslant 0.03$ for both SQUIDs.

All measurements are performed in a dilution refrigerator down to $20 \mathrm{mK}$. The setup is schematically shown in Fig. 1(a). The SQUIDs and gate are controlled by a combination of $\mathrm{dc}$ and rf signals, mixed together by bias tees. The rf signals are generated by synchronized arbitrary wave-form generators (AWG in the figure), guided to the sample by coaxial lines, attenuated and thermalized at different temperature stages. Low-pass filters (LP) with $60 \mathrm{~dB}$ attenuation up to $40 \mathrm{GHz}$ are placed at the sample stage. The dc wiring consists of $160 \Omega$ surface-mount resistors, 2-m-long lossy coaxial lines, and 1-m-long twisted pairs. At room temperature, a voltage bias $V_{b}$ produced by a floating digital-to-analog converter (DAC) is applied through a divider, and current is read out by a transconductance amplifier with a sensitivity $10^{-10} \mathrm{~A} / \mathrm{V}$. The sample is protected by two nested rf-tight shields in order to prevent microwave irradiation from higher-temperature stages.

We measure the sluice in the supercurrent branch, close to $V_{b}=0 . V_{b}$ is nulled by minimizing the current flowing without applying any pulses, thus compensating for the voltage induced by the current amplifier. Application of the flux 


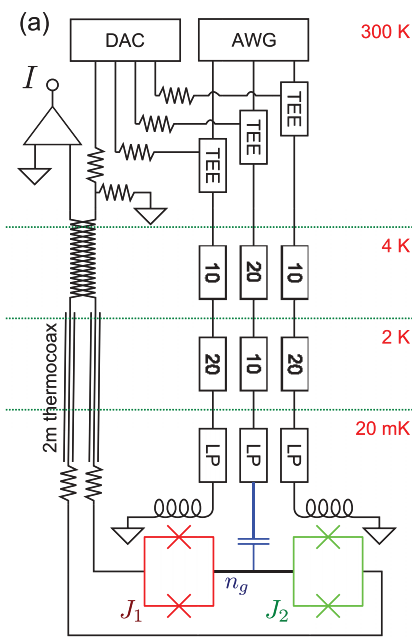

(b)

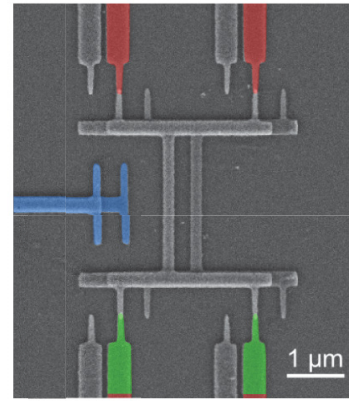

(c)

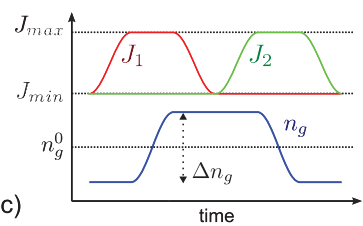

FIG. 1. (Color online) The Cooper-pair sluice. (a) Equivalent circuit of the sluice and scheme of the measurement setup. (b) False-color scanning electron micrograph of a representative device. (c) Time evolution of the control parameters $J_{1}, J_{2}$, and $n_{g}$ for a typical pumping cycle. pulses alone was found not to shift the zero-bias point, nor induce any additional current. This rules out the presence of rectification effects, which could have been introduced, e.g., by capacitive couplings between the on-chip coils and the leads. To further reduce the influence of voltage fluctuations, the pumped current $I_{p}$ is detected as the difference between the currents measured while pumping in opposite directions.

Evidence of single Cooper-pair pumping is presented in Fig. 2, where the pumped charge per cycle $Q_{p}=I_{p} / f$ ( $f$ is the pumping frequency) is plotted versus the offset $n_{g}^{0}$ and

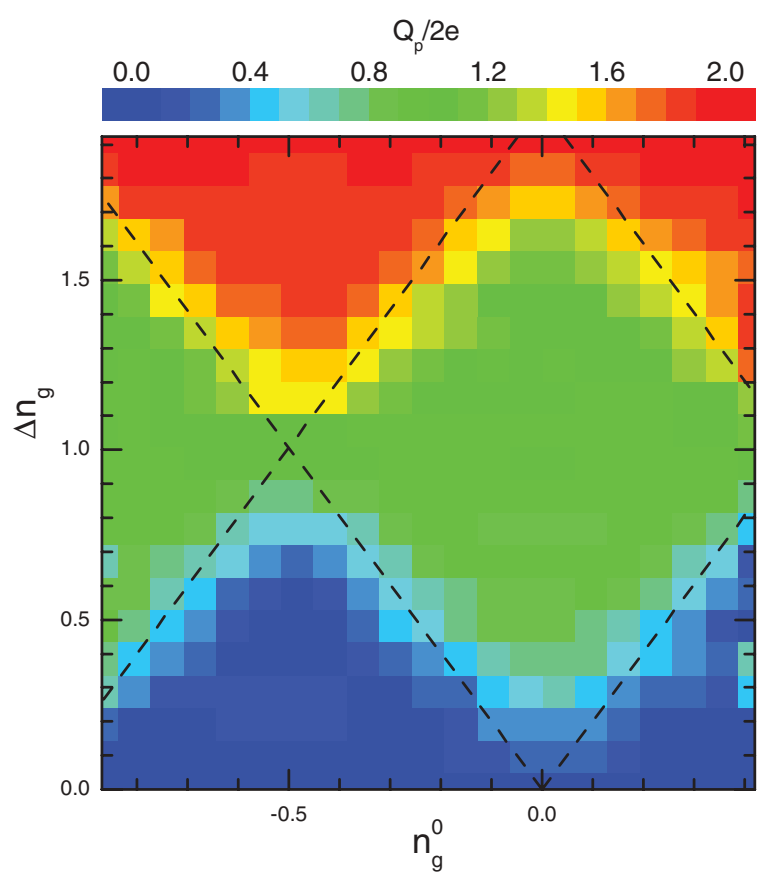

FIG. 2. (Color online) Single Cooper-pair pumping. Pumped charge $Q_{p}$ vs peak-to-peak amplitude $\Delta n_{g}$ and offset $n_{g}^{0}$ of the gate drive. The data are taken at zero bias voltage $V_{b}$. The pumping frequency is $f=80 \mathrm{MHz}$. The flux pulses are tuned so that $J_{\min }=$ $J_{\min }^{0}$ and $J_{\max }=0.8 J_{\max }^{0}$. Dashed lines enclose the diamond-shaped regions where $Q_{p} / 2 e$ is expected to be constant and quantized (assuming ideal operation). the peak-to-peak amplitude $\Delta n_{g}$ of the gate drive. We set $f=80 \mathrm{MHz}$, and tune the flux pulses [see Fig. 1(b)] so that $J_{\min }=J_{\min }^{0}$ and $J_{\max }=0.8 J_{\max }^{0}$. This choice of parameters ensures that the pumping cycle of Fig. 2 is fully adiabatic, as the rate of change of the adiabatic energy levels is always slow with respect to the instantaneous energy gap (for a discussion on the breakdown of the adiabatic theorem, see the following). As a result, we expect $Q_{p}$ to be quantized in units of Cooper pairs, the first correction being of the order of $J_{\min } / J_{\max } \cdot{ }^{13}$ From energy-diagram considerations, it is easy to show that the regions of constant $Q_{p}$ are diamond shaped in the offsetamplitude plane. The regions are delimited by the family of curves $\Delta n_{g}=2\left|n_{g}^{0}+m\right|$, where $m$ can be any integer.

A remarkable feature of Fig. 2 is that $Q_{p}$ is $2 e$ periodic in the gate charge (that is, the size of the diamonds is one unit along the $n_{g}^{0}$ and two units along the $\Delta n_{g}$ axis). Previous measurements with the Cooper-pair sluice ${ }^{17,19}$ reported $1 e$ periodic plateaus in the pumped charge plotted against the amplitude of the gate drive (no dependence on the gate offset was reported). The authors ascribed the observed periodicity to quasiparticle poisoning. Quasiparticle poisoning ${ }^{20}$ has been intensively studied in systems closely related to the sluice, the single Cooper-pair transistor ${ }^{21-23}$ and the single Cooper-pair box. $^{24,25}$ All these devices feature a superconducting island in the Coulomb-blockade regime. For a given position of the gate, there are two metastable states for the island ("odd state" and "even state"), differing by the presence of one quasiparticle. Nonequilibrium quasiparticles generated in the leads (at temperatures $T \ll \Delta / k_{B}$, the thermal population of quasiparticle states is unimportant) drive transitions between the two states ("parity fluctuations"), shifting the gate charge by exactly half a Cooper pair. In the sluice, this results in a $1 e$-periodic pumped charge, provided that the time scale of parity fluctuations is intermediate between the pumping period and the acquisition time. ${ }^{17,19}$ In our case, the clean $2 e$ periodicity observed implies that the device is not "poisoned" by quasiparticles. We ascribe this improvement to two factors: efficient microwave shielding, and careful avoidance of voltage fluctuations across the probe leads (we accomplished the latter by using a dedicated ground potential for the voltage source and the ammeter). The importance of microwave 
(a)
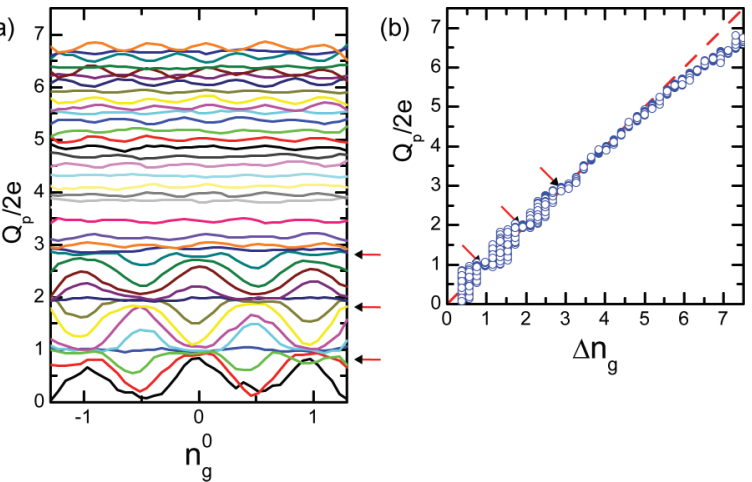

FIG. 3. (Color online) Crossover between pure Cooper-pair and mixed Cooper-pair-quasiparticle dynamics. (a) Pumped charge $Q_{p}$ vs gate offset $n_{g}^{0}$ for increasing gate amplitudes $\Delta n_{g}$ (bottom to top). Bias voltage and other pumping parameters are the same as in Fig. 2. (b) Circles: Same data as in (a), projected on the $n_{g}^{0}$ axis and plotted vs $\Delta n_{g}$. Dashed line: Asymptotic adiabatic-limit expectation. In both panels, the first three pumping plateaus are indicated by arrows.

shielding has been emphasized in other recent experimental works. ${ }^{26,27}$

While the data of Fig. 2 are clearly not affected by nonequilibrium quasiparticles, the latter may come into play as a result of nonadiabatic pumping. One way of making the pumping nonadiabatic is to increase the amplitude of the gate modulation. This increases both the effective speed of the drive (continuously), and the number of tunneling events involved (discretely). We do it in Fig. 3(a), where $Q_{p}$ is plotted versus $n_{g}^{0}$ with $\Delta n_{g}$ taking a series of values in the range of 0.1 and 7 . The same data are also plotted against $\Delta n_{g}$ in Fig. 3(b). The data show a clear crossover between pure Cooper-pair and mixed Cooper-pair-quasiparticle dynamics. Up to about $\Delta n_{g}=3$, $Q_{p}$ is $2 e$ periodic in $n_{g}^{0}$, as in Fig. 2. The pumping plateaus are also $2 e$ periodic in $\Delta n_{g}$. They show up as straight lines in Fig. 3(a), and nodes in Fig. 3(b). The first three plateaus are indicated by arrows. The crossover takes place between about $\Delta n_{g}=3$ and $\Delta n_{g}=5$, where the pattern is blurred. Finally, for $\Delta n_{g} \gtrsim 5$ a clear periodicity is restored, but the period has doubled. These data show that quasiparticle poisoning, while initially absent, can be induced by nonequilibrium quasiparticles generated by a nonadiabatic drive. The link between loss of adiabaticity and quasiparticle poisoning is strengthened by the fact that the crossover is accompanied by a reduction in $Q_{p}$ with respect to the adiabatic-limit expectation, as shown in Fig. 3(b) for $\Delta n_{g} \gtrsim 5$. As nonadiabatic transitions occur in the sluice as missed Cooper-pair tunnelings, they leave a detectable trace in overall magnitude of the pumped charge.

We understand the generation of nonequilibrium quasiparticles as a multistep process: LZT to an excited state followed by relaxation via Cooper-pair breaking and quasiparticle tunneling. When the drive is nonadiabatic, one or more Cooper pairs may fail to tunnel as dictated by the gate, leaving the island in an excited state. Even if there are no quasiparticles in the leads near the junctions, quasiparticle tunneling from or onto the island is still possible provided the energy gain of the process $\Delta E^{ \pm}\left[n, n_{g}\right]=4 E_{C}\left[\left(n-n_{g}\right)^{2}-\left(n-n_{g} \mp 1 / 2\right)^{2}\right]$ ( $n$ is the number of excess Cooper pairs on the island) exceeds the energy cost $2 \Delta$ required to break a Cooper pair. In our case, due to the moderate charging energy of our device $\left(E_{C}=0.33 \Delta\right)$, this is possible when the charge occupation of the island differs from that of the ground state by at least two Cooper pairs. After the tunneling of a quasiparticle, the island is left in a metastable state with an odd quasiparticle number, which may then decay by tunneling of a second quasiparticle. Overall, this mechanism is similar to the well-known Josephson-quasiparticle cycle, ${ }^{28}$ with the nonadiabatic gate drive playing the role of an effective voltage bias in dynamically creating nonequilibrium.

We now take a closer look at individual LZTs. To do so, we choose to pump a single Cooper pair at a time. This reduces the dynamics to that of a two-level system. Transitions between the adiabatic ground $(g)$ and excited state $(e)$ may occur due to Landau-Zener tunneling at avoided crossings [see Fig. 4(a)].

(a)
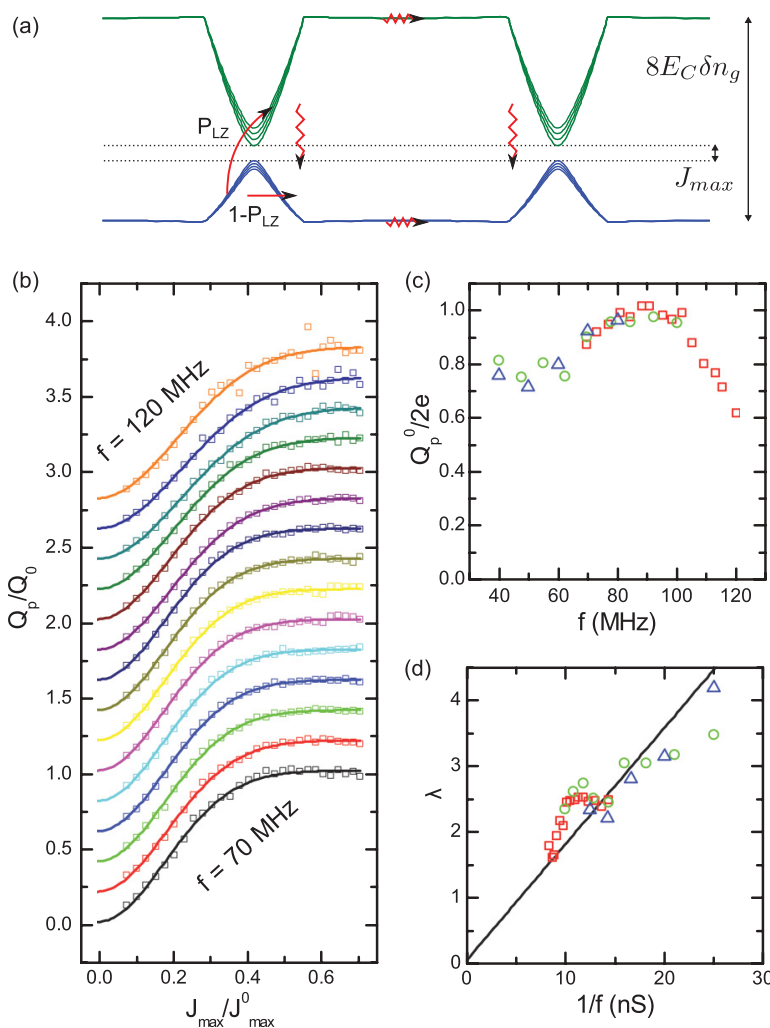

FIG. 4. (Color online) Nonadiabatic pumping and Landau-Zener transitions. (a) Instantaneous energies of the ground (bottom set of curves) and first excited state (top set) vs time for a pumping period (the two sets of curves are obtained by modulating the parameter $J_{\max }$ ). In the model described in the text, nonadiabatic transitions are localized at level crossings, and occur with probability $P_{\mathrm{LZ}}$. Decoherence induces complete dephasing between subsequent transitions, and relaxation via inelastic Cooper-pair tunneling (both are indicated by wavy arrows). (b) Normalized pumped charge $Q_{p} / Q_{0}$ vs Josephson coupling $J_{\max }$, for a set of frequencies in the range of 70 and $120 \mathrm{MHz}$. The gate pulse parameters are $n_{g}^{0}=0$ and $\Delta n_{g}=0.45$. The experimental traces (squares) are vertically offset by 0.2 , and plotted together with the best fit of Eq. (1) to them (solid lines). (c), (d) Asymptotic pumped charge $Q_{0}$ vs $f(\mathrm{c})$ and $\lambda$ parameter vs $1 / f(\mathrm{~d})$ for three different measurement sets (squares, circles, and triangles). The solid line in (d) is a fit to the data of the expression $\lambda=f_{0} / f$. 
For a single crossing, the transition probability $P_{\mathrm{LZ}}=e^{-2 \pi \delta}$ is governed by the adiabatic parameter $\delta=J_{\max }^{2} / \hbar v$, where $J_{\max }$ is the Josephson coupling of the active SQUID at the crossing and $v=E_{C} d n_{g} / d t$ the rate of change of the energy difference between diabatic (charge) states. The full tunability of our device gives us the possibility to control the degree of adiabaticity in several independent ways. In Fig. 4(b), we plot $Q_{p}$ vs $J_{\max }$, for the case $n_{g}^{0}=0$ and $\Delta n_{g}=0.45$. The traces are taken at different pumping frequencies in the range of 70 and $120 \mathrm{MHz}$, normalized to the asymptotic pumped charge $Q_{0}$, and vertically offset for clarity. All traces approach a constant value for large values of $J_{\max }$, and monotonically decrease to 0 for $J_{\max } / J_{\max }^{0} \rightarrow 0$.

In order to understand the data quantitatively, we must consider the interplay between LZTs and environment-induced decoherence. Decoherence effects in the sluice have been studied theoretically with a master-equation approach. ${ }^{18,29-32}$ Numerical calculations indicate that even in the presence of modest decoherence, the driven Cooper-pair sluice approaches a quasistationary state ${ }^{30}$ after a few tens of cycles. Since our acquisition time extends over about $10^{6}$ pumping cycles, we identify $Q_{p}$ with the quasistationary charge pumped by the sluice in the presence of its electromagnetic environment, averaged over a great number of cycles.

Let us now discuss the role of decoherence in determining the quasistationary state and hence $Q_{p}$. Due to the direct coupling to room-tempeture leads in the present design, we expect for the sluice a dephasing time similar to that of the Cooper-pair box with probe junctions, that is, of the order of a few ns. ${ }^{33}$ This implies that subsequent LZTs are totally uncorrelated, which rules out the possibility of Landau-ZenerStückelberg interference. ${ }^{34}$ The latter might instead play a role at higher pumping frequencies and/or in a closed-loop geometry. ${ }^{16}$ Besides dephasing, we also have to consider relaxation, which can take place via environment-assisted (inelastic) Cooper-pair tunneling through the SQUIDs. The energy scales and base temperature for our system are such that the transition rates to the excited state are exponentially suppressed, so that the environment is effectively at zero temperature. As a result, relaxation tends to keep the sluice in the ground state, ${ }^{18}$ counteracting LZTs. In general, determining the explicit dependence of $Q_{p}$ on $P_{\mathrm{LZ}}$ for an arbitrary environment is a formidable task, which can only be undertaken using numerical methods. In order to provide a simple analytical formula to be compared to our data, we shall further assume that (i) relaxation fully takes place between subsequent anticrossings, so that the system approaches every anticrossing in the ground state, and (ii) it takes place at the two anticrossings equally, so that on average it does not contribute to the pumped charge. Under (i) and (ii), the problem greatly simplifies, and we find that $Q_{p} / 2 e=1-P_{\mathrm{LZ}}$.
Based on this model, in Fig. 4(a) we fit to each experimental trace the expression

$$
Q_{p}(x)=Q_{0}\left[1-\exp \left(-2 \pi \lambda x^{2}\right)\right],
$$

where $x=J_{\max } / J_{\max }^{0}$ and $\lambda$ is a free parameter. The excellent agreement between the data and our single-parameter fit provides strong evidence that the departure from the adiabatic limit takes place via LZTs, and that our understanding of decoherence effects, albeit simplified, is essentially correct.

The dependence of $Q_{0}$ and $\lambda$ on $f$ is shown in Figs. 4(c) and 4(d). Different symbols refer to three different measurement sets, taken on the same sample using different generators and/or during different cooldowns. Altogether, they span the frequency range of 40 and $120 \mathrm{MHz}$. This range is limited from below by an acceptable signal-to-noise ratio, and from above by the sampling rate of our rf generators. As the three sets yield consistent results, we discuss them together. The behavior of $Q_{0}$ [Fig. 4(c)] is not clear; it may stem from frequency-dependent attenuation in the lines. By contrast, $\lambda$ [Fig. 4(d)] displays a clear trend, a steady increase with decreasing $f$. The solid line in Fig. 4(d) is a best fit to the data of the expression $\lambda=f_{0} / f$, yielding $f_{0}=180 \mathrm{MHz}$. Our model predicts $f_{0}^{\text {(th) }}=\left(J_{\text {max }}^{0}\right)^{2} /\left(4 \hbar \alpha E_{C} \Delta n_{g}\right)$, where $\alpha \approx 4.3$ is a parameter proportional to the steepness of the gate rise. Using our estimated $E_{C}$ and $J_{\max }^{0}$, we obtain $f_{0}^{\text {(th) }} \approx 120 \mathrm{MHz}$. We find the agreement between $f_{0}$ and $f_{0}^{\text {(th) }}$ to be satisfactory. The discrepancy between the two corresponds to an effective Josephson energy about 20 smaller than its nominal value. Among other factors, this may well be due to decoherence effects not captured by our simple model.

Our results demonstrate the feasibility of using Cooper-pair pumps to investigate coherent effects in driven quantum systems, and pave the way for further observations. By embedding the sluice in a fully superconducting loop (as, e.g., in Refs. 15 and 35) and increasing the pumping frequency up to a few hundred $\mathrm{MHz}$, we expect to achieve coherence times extending over several pumping periods. This would allow challenging proposals such as Landau-Zener interferometry with geometric phases, ${ }^{16}$ characterization of decoherence induced by an engineered environment, ${ }^{29}$ and measurement of the Lamb shift, ${ }^{36}$ to be readily implemented.

We would like to thank V. Maisi, M. Möttönen, and O.-P. Saira for useful discussions, and T. Faivre and M. Meschke for technical assistance. This work was supported by the European Community FP7 under Grants No. 238345 "GEOMDISS" and No. 228464 "MICROKELVIN," by the Academy of Finland through its Centers of Excellence Program (Project No. 251748), and by the Finnish National Graduate School in Nanoscience. *simone.gasparinetti@aalto.fi

${ }^{1}$ M. D. Blumenthal, B. Kaestner, L. Li, S. P. Giblin, T. J. B. M. Janssen, M. Pepper, D. Anderson, G. A. C. Jones, and D. A. Ritchie, Nat. Phys. 3, 343 (2007).
${ }^{2}$ S. P. Giblin, S. J. Wright, J. D. Fletcher, M. Kataoka, M. Pepper, T. J. B. M. Janssen, D. A. Ritchie, C. A. Nicoll, D. Anderson, and G. A. C. Jones, New J. Phys. 12, 073013 (2010). 
${ }^{3}$ F. Giazotto, P. Spathis, S. Roddaro, S. Biswas, F. Taddei, M. Governale, and L. Sorba, Nat. Phys. 7, 857 (2011).

${ }^{4}$ L. Nevou, V. Liverini, P. Friedli, F. Castellano, A. Bismuto, H. Sigg,

F. Gramm, E. Müller, and J. Faist, Nat. Phys. 7, 423 (2011).

${ }^{5}$ S. P. Giblin, M. Kataoka, J. D. Fletcher, P. See, T. Janssen, J. P. Griffiths, G. A. C. Jones, I. Farrer, and D. A. Ritchie, Nat. Commun. 3, 930 (2012).

${ }^{6}$ J. P. Pekola, J. J. Vartiainen, M. Möttönen, O.-P. Saira, M. Meschke, and D. V. Averin, Nat. Phys. 4, 120 (2008).

${ }^{7}$ G. Fève, A. Mahé, J.-M. Berroir, T. Kontos, B. Plaçais, D. C. Glattli, A. Cavanna, B. Etienne, and Y. Jin, Science 316, 1169 (2007).

${ }^{8}$ J. Flowers, Science 306, 1324 (2004).

${ }^{9}$ J. P. Pekola, J. J. Toppari, M. Aunola, M. T. Savolainen, and D. V. Averin, Phys. Rev. B, 60, R9931 (1999).

${ }^{10}$ A. O. Niskanen, J. P. Pekola, and H. Seppä, Phys. Rev. Lett. 91, 177003 (2003).

${ }^{11}$ R. Leone, L. P. Lévy, and P. Lafarge, Phys. Rev. Lett. 100, 117001 (2008).

${ }^{12}$ F. Hoehne, Y. A. Pashkin, O. V. Astafiev, M. Möttönen, J. P. Pekola, and J. S. Tsai, Phys. Rev. B 85, 140504 (2012).

${ }^{13}$ M. Möttönen, J. P. Pekola, J. J. Vartiainen, V. Brosco, and F. W. J. Hekking, Phys. Rev. B 73, 214523 (2006).

${ }^{14}$ M. Aunola and J. J. Toppari, Phys. Rev. B 68, 020502(R) (2003).

${ }^{15}$ M. Möttönen, J. J. Vartiainen, and J. P. Pekola, Phys. Rev. Lett. 100, 177201 (2008).

${ }^{16}$ S. Gasparinetti, P. Solinas, and J. P. Pekola, Phys. Rev. Lett. 107, 207002 (2011).

${ }^{17}$ A. O. Niskanen, J. M. Kivioja, H. Seppä, and J. P. Pekola, Phys. Rev. B 71, 012513 (2005).

${ }^{18}$ J. P. Pekola, V. Brosco, M. Möttönen, P. Solinas, and A. Shnirman, Phys. Rev. Lett. 105, 030401 (2010).

${ }^{19}$ J. J. Vartiainen, M. Möttönen, J. P. Pekola, and A. Kemppinen, Appl. Phys. Lett. 90, 082102 (2007).
${ }^{20}$ P. Joyez, P. Lafarge, A. Filipe, D. Esteve, and M. H. Devoret, Phys. Rev. Lett. 72, 2458 (1994).

${ }^{21}$ J. Aumentado, M. W. Keller, J. M. Martinis, and M. H. Devoret, Phys. Rev. Lett. 92, 066802 (2004).

${ }^{22}$ O. Naaman and J. Aumentado, Phys. Rev. B 73, 172504 (2006).

${ }^{23}$ A. J. Ferguson, N. A. Court, F. E. Hudson, and R. G. Clark, Phys. Rev. Lett. 97, 106603 (2006).

${ }^{24}$ M. D. Shaw, R. M. Lutchyn, P. Delsing, and P. M. Echternach, Phys. Rev. B 78, 024503 (2008).

${ }^{25}$ F. Persson, C. M. Wilson, M. Sandberg, and P. Delsing, Phys. Rev. B 82, 134533 (2010).

${ }^{26}$ R. Barends, J. Wenner, M. Lenander, Y. Chen, R. C. Bialczak, J. Kelly, E. Lucero, P. O’Malley, M. Mariantoni, D. Sank, H. Wang, T. C. White, Y. Yin, J. Zhao, A. N. Cleland, J. M. Martinis, and J. J. A. Baselmans, Appl. Phys. Lett. 99, 113507 (2011).

${ }^{27}$ O.-P. Saira, A. Kemppinen, V. F. Maisi, and J. P. Pekola, Phys. Rev. B 85, 012504 (2012).

${ }^{28}$ T. A. Fulton, P. L. Gammel, D. J. Bishop, L. N. Dunkleberger, and G. J. Dolan, Phys. Rev. Lett. 63, 1307 (1989).

${ }^{29}$ P. Solinas, M. Möttönen, J. Salmilehto, and J. P. Pekola, Phys. Rev. B 82, 134517 (2010).

${ }^{30}$ A. Russomanno, S. Pugnetti, V. Brosco, and R. Fazio, Phys. Rev. B 83, 214508 (2011).

${ }^{31}$ J. Salmilehto and M. Möttönen, Phys. Rev. B 84, 174507 (2011).

${ }^{32}$ I. Kamleitner and A. Shnirman, Phys. Rev. B 84, 235140 (2011).

${ }^{33}$ Y. Nakamura, Y. A. Pashkin, and J. S. Tsai, Nature (London) 398 , 786 (1999).

${ }^{34}$ S. N. Shevchenko, S. Ashhab, and F. Nori, Phys. Rep. 492, 1 (2010).

${ }^{35}$ D. Vion, A. Aassime, A. Cottet, P. Joyez, H. Pothier, C. Urbina, D. Esteve, and M. H. Devoret, Science 296, 886 (2002).

${ }^{36}$ V. Gramich, P. Solinas, M. Möttönen, J. P. Pekola, and J. Ankerhold, Phys. Rev. A 84, 052103 (2011). 